P193 (continued)

eat more vegetables as a result of the activity. Plans to eat more vegetables include: preparing more salads (38\%); making the recipe (14\%); buying more vegetables $(22 \%)$, and none because they were eating enough (28\%). Participants comments were also good "it taste good and fast to do" "simple and delicious". This activity increased the wiliness of participants to eat, prepare and buying more vegetables. This program is funded by USDA-SNAP-Ed program.

Funding: Supplemental Nutrition Assistance Program Education

\section{P194 WIC Nutrition Education is Evolving to Address Value Enhanced Nutrition Education (VENA) Guidance}

Sheryl Cates, BA, RTI International; Kristen C. Giombi, PhD, kcapogrossi@rti.org, RTI International, 3040 East Cornwallis Road, PO Box 12194, Research Triangle Park, NC 27709; Linnea Sallack, MPH, RD, Altarum Institute; Karen Deehy, MS, RD; Karen Castellanos-Brown, PhD, MSW, USDA, Food and Nutrition Service

Objective: In 2006, the Food and Nutrition Service introduced the Value Enhanced Nutrition Assessment (VENA) guidance to aid with participant-centered nutrition education as part of the WIC program. Because local WIC agencies have flexibility in their approach for delivering nutrition education, understanding how current practices align with VENA was an objective of the 2016 WIC Nutrition Education Study: Phase I Report.

Study Design, Setting, Participants, Intervention: We conducted surveys with a nationally representative sample of WIC local agencies and sites (893 local agencies, 1,401 sites) and in-depth telephone interviews with site staff from a subset of survey respondents (80).

Outcome Measures and Analysis: Outcome measures included environment, staffing, and resources for WIC nutrition education; types and frequency of educational approaches; and efforts to incorporate participantcentered approaches. We synthesized data from surveys and interviews.

Results: Up to $67 \%$ of sites provide staff training on VENA and/or participant-centered education techniques with an average of 5-6 hours of training per staff person annually. Sites reporting more training also reported more changes in their nutrition education approach. More than $80 \%$ of sites indicated that discussion topics for one-on-one visits are chosen by the participant or by the participant and WIC staff together. Similar numbers reported that individualized goals are almost always or often set by participants or by the participant and educator together.

Conclusions and Implications: WIC nutrition education is moving toward a more individualized approach focused on meeting participant needs with a higher level of participant involvement throughout the process which is the primary goal of VENA. Ongoing attention to strengthening nutrition education policy and practice is essential for achieving program goals.

Funding: USDA

\section{P195 Farm Fresh Food Boxes: Increasing Food Access in Rural Communities through New Markets for Farmers and Retailers}

Diane Smith, MA, diane.smith@wsu.edu, Washington State University Extension, 11768 Westar Lane, Suite A, Burlington, WA 98233; Florence Becot, MS, University of Vermont Extension; Lisa Chase, PhD; Hans Estrin, MS; Lauren Greco, BS; Jane Kolodinsky, PhD; Erin Roche, MS; Marilyn Sitaker, MPH, Evergreen State College; Julia Van Soleon Kim, MPH, MS, University of California Extension

Objective: Farm Fresh Food Boxes (FFFB) is an integrated research and extension project that takes an innovative an innovative approach to make healthy, affordable food available in food deserts by connecting farmers, retailers, and consumers.

Study Design and Participants: The FFFB project is a multi-state collaboration. The extension team engages farms and associated retail sites in three participating states: Vermont, Washington, and California, and promotes this innovative program to consumers in rural communities with limited access to fresh, local produce. The research team conducts mixedmethods research on the economic impacts of FFFB on farmers, retailers, and consumers. Participants were farmers, rural community members, and small food retailers.

Intervention: Farms offer weekly boxes of fresh produce at retail sites that provide convenient access to consumers. Retail sites post flyers detailing the content and cost of FFFB from participating area farms. Customers' pre-order advertised boxes at the retail site or online on a week-to-week basis for later pick-up. Box contents and flyers change throughout the season to move produce that is most abundant.

Outcome Measures and Analysis: A mixed-methods research protocol will assess the economic impacts on farmers, retailers, and consumers using surveys and interviews.

Results: Preliminary data reflects project outcomes which include: market potential, measure of economic impact, measure of acceptability, comparative use of FFFB among consumer at Farmers Market and CSA shares, and benefits and barriers to farmers, retailers, and consumers.

Conclusions and Implications: FFFB provides a lowrisk strategy to address the complex supply, demand, and distribution challenges faced by producers and retailers of fresh local foods, while overcoming barriers that consumers face in accessing affordable, healthy food.

Funding: NIFA 